\section{Identifying the source of tumour-infiltrating $T$ cells}

\author{
Suman Kumar Vodnala \& Nicholas P. Restifo
}

Immune cells called cytotoxic T cells can recognize and

destroy cancer cells. The finding that stem-cell-like T cells

exist in tumours, at niche sites that support these cells, could aid efforts to boost anticancer immune responses. See p.465

Certain anticancer treatments have been revolutionized by the ability to harness a person's own immune cells for therapeutic purposes ${ }^{1}$. Such immunotherapy can result in lasting anticancer responses in people with advanced-stage blood cancers or solid tumours. But not everyone responds. For a variety of cancers, the presence of cytotoxic T cells - immune cells that can kill cancer cells - in a tumour correlates with, but does not predict, an anticancer response and survival. And it is unclear why robust tumour infiltration by T cells occurs in some people, but not in others. On page 465, Jansen et al. ${ }^{2}$ reveal a previously unknown source of tumour-infiltrating T cells.

Because tumour cells can proliferate continuously, tumour-targeting T cells must have a similar ability to persist and divide until the last remaining tumour cell is eradicated. In people undergoing immunotherapy, a greater longevity of antitumour T cells correlates with a better therapeutic outcome ${ }^{3}$. Therefore, for effective immunotherapy, it is crucial to understand the factors that influence T-cell persistence and infiltration of tumours. Some clues already exist ${ }^{4}$ about these factors, such as the presence of long versions of structures called telomeres, found at the ends of chromosomes, and high expression levels of the protein CD27 in T cells.

In addition to these factors, another clue came from the identification of a subset of stem-cell-like T cells called memory T cells, which can provide long-lasting immune responses $^{5,6}$, and which express high levels of TCF7 (previously known as TCF-1). This protein is important for maintaining a stemcell-like state in $\mathrm{T}$ cells that also express the protein CD8 (known as CD8 T cells) ${ }^{7,8}$. Such stem-cell-like cells can self-renew and give rise to different types of $T$ cell, including a type of CD8 $\mathrm{T}$ cell called a cytotoxic CD8 T cell. The presence of stem-cell-like T cells in people who have cancer was reported previously ${ }^{5}$; however, the anatomical location of these cells had not been elucidated. Jansen and colleagues now show that human kidney tumours contain stem-cell-like T cells that reside in the tumour in niches that support them (Fig. 1).

The authors investigated how the level of tumour-infiltrating cytotoxic CD8 T cells varied. They analysed samples of human kidney tumours obtained from people who had undergone tumour-removal surgery, and noted a wide variation in the level of T-cell infiltration between the samples. In people who had tumours in which CD8 T cells accounted for fewer than $2.2 \%$ of cells in the sample, the cancer continued to grow, indicating that the surgery and the person's immune response to the residual cancer cells were insufficient to halt disease progression. By contrast, above this threshold of $2.2 \%$ infiltration, cancer growth after surgery was four times slower.

Jansen and colleagues then studied the composition and type of $\mathrm{T}$ cell in the tumour samples using a technique called flow cytometry, and identified two distinct sets of T cell. One set consisted of cytotoxic CD8 T cells that express high levels of cancer-killing molecules but that also express 'immune-checkpoint' molecules. Expression of checkpoint molecules can drive cytotoxic T cells to enter a dysfunctional state known as exhaustion, which can occur in the tumour microenvironment after prolonged exposure to cancer cells recognized by the T cells. The
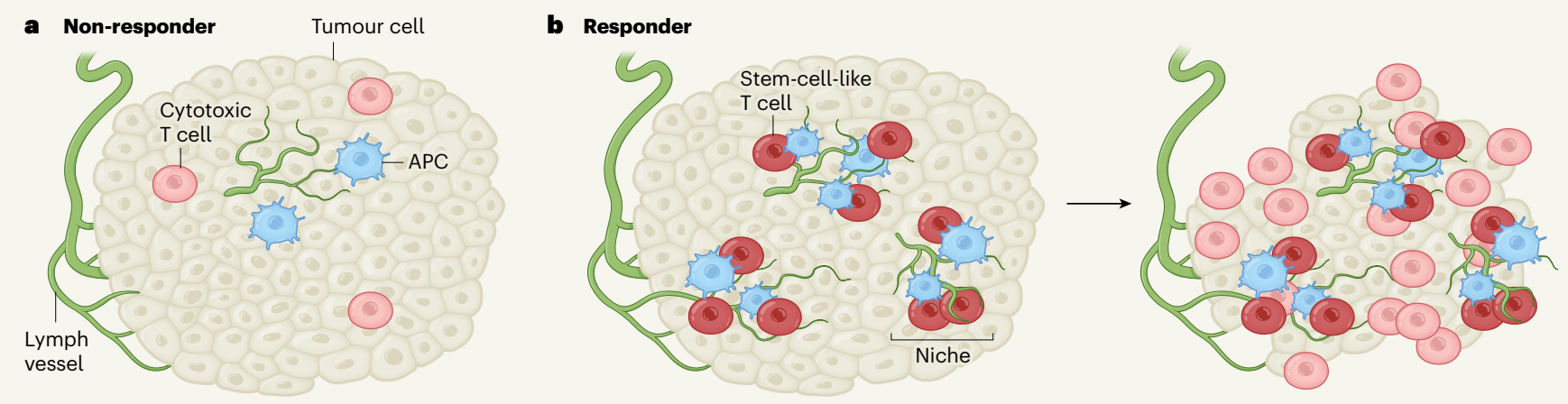

Figure 1 | Stem-cell-like T cells reside in niches in tumours. Jansen et al. ${ }^{2}$ report the discovery of stem-cell-like T cells that inhabit kidney tumours. a, The authors compared the profile of tumour samples obtained from people who had undergone tumour-removal surgery. In non-responders, whose cancer progressed more rapidly after surgery, there was a low level of tumour infiltration by cancer-killing (cytotoxic) T cells of the immune system. These tumours contain antigen-presenting cells (APCs) and structures called lymph vessels. b. By contrast, people who had longer progression-free survival after surgery had a high level of tumour infiltration by cytotoxic $\mathrm{T}$ cells. The authors report that the tumours of these responders had sites, called niches, containing stem-cell-like $T$ cells that could give rise to cytotoxic $T$ cells. These niches were associated with APCs and lymph vessels. There were more lymph vessels in these responding tumours than in the non-responding tumours. 
other set consisted of stem-cell-like $\mathrm{T}$ cells that Jansen et al. demonstrate give rise to cytotoxic CD8 T cells that help to promote an effective antitumour immune response. Stem-cell-like T cells were present only at very low levels in tumours with low levels of T-cell infiltration, whereas tumours with high levels of T-cell infiltration had high levels of the stem-cell-like T cells.

Togain further insight, the authors assessed cellular gene-expression profiles, and analysed epigenetic modifications - types of modification to DNA and its associated proteins that can affect gene expression. They found that, compared with the exhausted cytotoxic CD8 T cells, the stem-cell-like T cells express distinctive immune-signalling molecules called chemokines that are correlated with better patient survival, along with higher levels of key co-stimulatory molecules (which are essential for T-cell differentiation into cytotoxic T cells). Previous analyses ${ }^{9,10}$ of T cells revealed a pattern of progressive steps in epigenetic modification as stem-cell-like $\mathrm{T}$ cells give rise to cytotoxic CD8 T cells and then eventually become exhausted.

The epigenetic-modification profile of $T$ cells in tumours can be profoundly influenced by factors in the tumour microenvironment, which can affect the ability of T cells to function as stem cells ${ }^{11,12}$. For example, the concentration of potassium ions in a tumour modulates epigenetic modifications that influence whether T cells are in the stemcell-like state that is needed for them to give rise to cytotoxic CD8 T cells ${ }^{11,12}$. The effect of the tumour microenvironment on the development of cancer-targeting $T$ cells is unclear, and should be a subject for future studies.

Jansen and colleagues noted that the higher than normal expression of chemokines and chemokine-binding receptors in the stemcell-like T cells is similar to that seen in cells in the microenvironment of lymph vessels structures through which immune cells move and which support T-cell activation and survival. The authors' analyses demonstrate that stem-cell-like T cells are located in niches in tumours near lymph vessels (Fig. 1), and are confined to dense zones of antigen-presenting cells, which can prime T cells to target tumours. The discovery of these niches by Jansen and colleagues now reveal how stemcell-like $T$ cells can be maintained in tumours in a functional state capable of generating cytotoxic T cells.

The authors observed a correlation between the presence of protein markers of stem-celllike T-cell niches and longer, progression-free survival of the people assessed in the study. By contrast, other common ways of assessing an immune response in tumours, such as the expression of the immune-checkpoint protein PD-L1, did not reveal a correlation with progression-free cancer survival.
Previous research ${ }^{13}$ identified stemcell-like $T$ cells that express rising levels of immune-checkpoint molecules as they progress towards forming cytotoxic CD8 T cells that eventually become exhausted ${ }^{14}$. In one example ${ }^{13}$, approaches to block the immune-checkpoint protein PD-1 caused a burst of proliferation in stem-cell-like T cells that express the TCF7 protein. Similarly, in a skin cancer called melanoma, people whose CD8 T cells express TCF7 have a better clinical outcome if they receive immunotherapy to block immune-checkpoint proteins ${ }^{15}$. These results suggest that people whose tumours cannot be removed by surgery might benefit from therapy that blocks immune-checkpoint molecules, if their tumours contain stem-cell-like T cells.

Jansen and colleagues' work raises questions about how the stem-cell niches are generated and maintained, and whether tumours might act on them to evade destruction by the immune system. The discovery that resident stem-cell-like T cells exist in specialized niches in tumours suggests that clinical leveraging of such cells to increase the immune infiltration of tumours, together with immunotherapy to boost exhausted T cells, might unleash T-cell responses to aid the success of anticancer treatment.

\section{Suman Kumar Vodnala and Nicholas}

P. Restifo are at Lyell Immunopharma, South San Francisco, California 94080, USA. e-mail: nrestifo@lyell.com

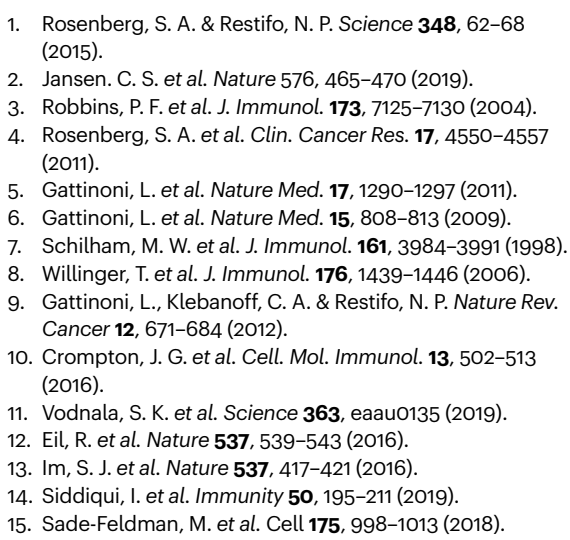

1. Rosenberg, S. A. \& Restifo, N. P. Science $\mathbf{3 4 8 , 6 2 - 6 8}$ (2015).

2. Jansen. C. S. et al. Nature 576, 465-470 (2019).

3. Robbins, P. F. et al. J. Immunol. 173, 7125-7130 (2004).

4. Rosenberg, S. A. et al. Clin. Cancer Res. 17, 4550-4557 (2011).

5. Gattinoni, L. et al. Nature Med. 17, 1290-1297 (2011).

6. Gattinoni, L. et al. Nature Med. 15, 808-813 (2009).

7. Schilham, M. W. et al. J. Immunol. 161, 3984-3991 (1998).

8. Willinger, T. et al. J. Immunol. 176, 1439-1446 (2006).

9. Gattinoni, L., Klebanoff, C. A. \& Restifo, N. P. Nature Rev. Cancer 12, 671-684 (2012).

10. Crompton, J. G. et al. Cell. Mol. Immunol. 13, 502-513 (2016).

11. Vodnala, S. K. et al. Science 363, eaau0135 (2019).

12. Eil, R. et al. Nature 537, 539-543 (2016).

13. Im, S. J. et al. Nature 537, 417-421 (2016)

14. Siddiqui, I. et al. Immunity 50, 195-211 (2019).

15. Sade-Feldman, M. et al. Cell 175, 998-1013 (2018).

The authors declare competing financial interests: see go.nature.com/2sivhan for details.

This article was published online on 11 December 2019.

\section{In Retrospect}

\section{Superconductivity mystery turns 25}

\section{N. Peter Armitage}

In 1994, an unconventional form of superconductivity was

detected in strontium ruthenate. The discovery has shed light

on the mechanism of unconventional superconductivity at high

temperatures.

Superconductivity is an effect in which a material's electrical resistance vanishes and any magnetic field is expelled below a transition temperature. Despite the remarkable phenomenology, this behaviour is actually quite common: almost half the elements in the periodic table are superconductors ${ }^{1}$, albeit at temperatures near or below the extremely low one at which helium gas liquefies (about 4 kelvin). Since Nobel-prizewinning work in the late 1950s, we have had a successful theory ${ }^{2}$ of superconductivity in these conventional systems. Electrons bind into 'Cooper pairs' that have isotropic (direction-independent) properties through an interaction with vibrations of surrounding ions. Over the past 40 years, researchers have looked for unconventional superconductors that involve different pairing interactions, such as magnetic ones. In 1994, Maeno et al. ${ }^{3}$ reported one of the clearest examples of unconventional superconductivity, in strontium ruthenate near $1 \mathrm{~K}$.

Understanding unconventional superconductors requires identifying both the pairing interaction and the order parameter - a quantity that reflects the interaction and the macroscopic, typically anisotropic, properties of the unconventional superconductivity. The most substantial development in this area of study was the discovery of superconductivity in layered copper-oxide compounds (known as cuprates) in the mid-to-late1980s. The phenomenon was detected ${ }^{4}$ at the unprecedentedly high temperature (for that time) of $30 \mathrm{~K}$, which led to a worldwide effort to understand the mechanism of cuprate superconductivity. 